\title{
A Model based Test Pattern Generation and Testing Framework for IoT Applications
}

\author{
V.Sathyavathy, D.Shanmuga Priyaa
}

\begin{abstract}
The whole world is entering towards the trend of smart technology. Internet of Things ( IoT) is an important domain behind this enormous growth. A simple IoT system consists of a device or actuators or sensors, which are connected to software with the help of an internet. The embedded sensors can be monitored and managed from remote place through the network from anywhere in the world internet. There are various applications that are supported in this domain due to this feature. They are smart agriculture, Home automation, been applied in various domains like Home automation, Patient Health Monitoring, Smart City, Smart Agriculture and much more. The usage of these applications is increasing day by day, so there arises a need for verifying and validating the IoT devices in all aspects.

The test automation framework that generates test pattern for various testing of IoT application domains that deploys in a sequence process of test patterns which can be easily started for the development of IoT scenarios described. To test their IoT device, there is a need for proper testing techniques for IoT applications through different IoT developers follow their own strategy. The main goal of the automation framework is to reduce the effort in the testing process and to make the test process easier for testing the IoT applications by generating various test patterns various tests depends on a number of IoT test patterns, which allows the process of various operations as the future extension.
\end{abstract}

Keywords: Test Pattern, Internet of Things, Test Model, Testing Framework

\section{INTRODUCTION}

The IoT network in future, the number of devices which is connected and services provided will be increased that will be reflected in a heterogeneity and diversity of embedded software, hardware platforms, network protocols, and service providers [1]. The realization of the IoT paradigm implies many challenges that need to be addressed, including availability, reliability, mobility, performance, scalability, interoperability, security and management. Healthcare is only one of the domains that will benefit from the vast range of solutions IoT can provide. There are number of faults that can be tested out by using several tools in the IoT that can be pointed out. With the knowledge that failures in IoT applications can have dire consequences, the importance of ensuring their correctness becomes apparent

\section{RELATED WORK}

- $\quad$ Identifying the exact use cases [2].

- $\quad$ Testing the application domains for various factors such as functionality, reliability, scalability, Performance, usability, security, and much more for it to be successful To address this issue, this work aimed to:

- Identify the exact use cases and test cases of existing test solutions

- Identify the short-comes of existing test solutions;

- Formulating an IoT framework and designing test patterns

Table 1: Consequences of Testing in IoT

IoT research in the field of healthcare has been focused on improving the quality of care through remote

\begin{tabular}{|l|l|}
\hline \multicolumn{1}{|c|}{ Issues } & \multicolumn{1}{c|}{ Consequences of Testing } \\
\hline $\begin{array}{l}\text { Testing for IoT } \\
\text { Solutions is specific }\end{array}$ & $\begin{array}{l}\text { To define efficient test strategy } \\
\text { for IoT Solutions is a difficult task }\end{array}$ \\
\hline $\begin{array}{l}\text { Security and privacy } \\
\text { threats }\end{array}$ & $\begin{array}{l}\text { For security testing and } \\
\text { authorization aspects there is an } \\
\text { increasing demand }\end{array}$ \\
\hline $\begin{array}{l}\text { Cost effective and } \\
\text { energy consumption }\end{array}$ & $\begin{array}{l}\text { More efficient methods and } \\
\text { techniques are in great demand to } \\
\text { select cost effective variants to } \\
\text { test }\end{array}$ \\
\hline $\begin{array}{l}\text { Industry standardized } \\
\text { IOT devices }\end{array}$ & $\begin{array}{l}\text { There is a need for more } \\
\text { automated integration testing, } \\
\text { that are possibly efficient }\end{array}$ \\
\hline $\begin{array}{l}\text { Demand rises for the } \\
\text { lower prices for the } \\
\text { IoT devices }\end{array}$ & $\begin{array}{l}\text { Both manual and automation } \\
\text { process are required to test the } \\
\text { number of variants }\end{array}$ \\
\hline $\begin{array}{l}\text { Ensuring the } \\
\text { reliability of service }\end{array}$ & $\begin{array}{l}\text { In edge testing multiple users } \\
\text { under limited connection needed } \\
\text { to be tested specifically for } \\
\text { life-critical systems }\end{array}$ \\
\hline
\end{tabular}

health monitoring solutions, there is already some work in the area of standards and protocols, presenting solutions designed for specific deployment scenarios.

Table 2: Deployment scenario in health care

\begin{tabular}{|c|c|c|c|}
\hline Project & $\begin{array}{c}\text { Deployment } \\
\text { Scenarios }\end{array}$ & Protocols & Standard \\
\hline
\end{tabular}


A Model based Test Pattern Generation and Testing Framework for IoT Applications

\begin{tabular}{|l|l|l|l|}
\hline $\begin{array}{l}\text { Abidoye, } \\
\text { AdemolaP }\end{array}$ & Home & $\begin{array}{l}\text { ZigBee/IEEE80 } \\
\text { 2.15.4 standard }\end{array}$ & $3 \mathrm{G}$ \\
\hline Yang Et al & $\begin{array}{l}\text { Home, } \\
\text { Hospital }\end{array}$ & $\begin{array}{l}\text { BlueTooth,Zig } \\
\text { Bee }\end{array}$ & N/A \\
\hline
\end{tabular}

\section{IoT TESTING TYPES}

\subsection{Testing the components of IoT}

Testing for IoT components generally comprises of Security, Protocol standards, Sensors, Network communication, Processors, Operating Systems and Platforms [3].

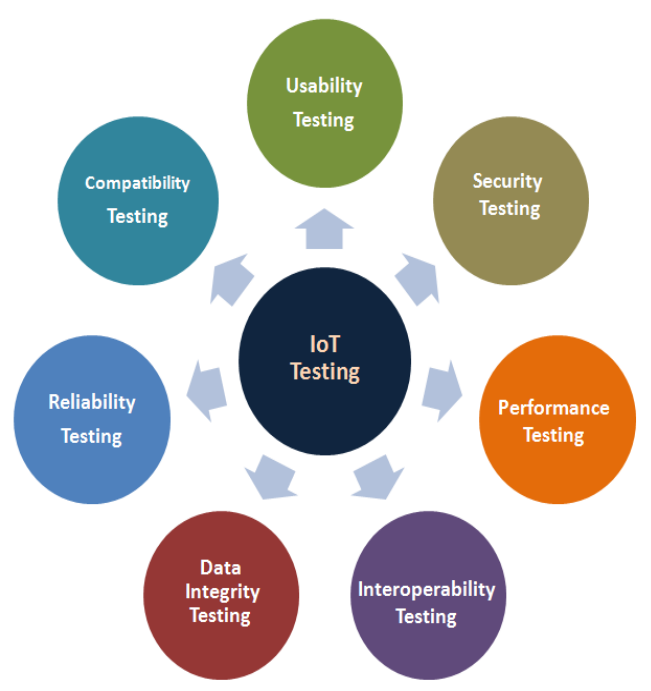

Figure 1: Testing Types of various components of IoT

As it's far a healthcare solution, connectivity plays an essential role. The device needs to accessible all of the time and to have endless connectivity with the stakeholders. Being a tester, there is a need to test the offline situations as properly. Once the device is not accessible at the network, there must be an alert that could set off the physicians in order to start to screen the fitness situations manually not relying on the device till it is up.

The following chart describes the applications of different types of testing for various devices of IOT.

Table 3.1a: Types of Testing in IoT

\begin{tabular}{|l|l|l|l|}
\hline \multicolumn{1}{|c|}{$\begin{array}{c}\text { Testing } \\
\text { Types }\end{array}$} & $\begin{array}{l}\text { Functional } \\
\text { Testing }\end{array}$ & $\begin{array}{l}\text { Usability } \\
\text { Testing }\end{array}$ & Security \\
\hline Sensor & True & True & True \\
\hline $\begin{array}{l}\text { Applicatio } \\
\mathrm{n}\end{array}$ & True & True & True \\
\hline Network & False & False & True \\
\hline Back End & False & False & True \\
\hline
\end{tabular}

Table 3.1b: Types of Testing in IoT

\begin{tabular}{|c|c|c|c|}
\hline $\begin{array}{l}\text { Testing } \\
\text { Types }\end{array}$ & $\begin{array}{c}\text { Performanc } \\
\text { e }\end{array}$ & Compatibility & $\begin{array}{c}\text { Service } \\
\mathrm{s}\end{array}$ \\
\hline Sensor & False & True & False \\
\hline Applicatio & True & True & True \\
\hline
\end{tabular}

\begin{tabular}{|l|l|l|l|}
\hline $\mathrm{n}$ & & & \\
\hline Network & True & False & True \\
\hline Back End & True & False & True \\
\hline
\end{tabular}

\begin{tabular}{|c|l|l|l|}
\hline Project & $\begin{array}{c}\text { Deployment } \\
\text { Scenario }\end{array}$ & Protocols & Standard \\
\hline Rahmani & $\begin{array}{l}\text { Home, } \\
\text { Pharmacy, } \\
\text { Nurrsing Home }\end{array}$ & $\begin{array}{l}\text { BlueTooth, } \\
\text { Wi-Fi }\end{array}$ & $\begin{array}{l}\text { IEEE } \\
11073\end{array}$ \\
\hline Sapnora & $\begin{array}{l}\text { Home, Nursing } \\
\text { Home }\end{array}$ & $\begin{array}{l}\text { BLE, } \\
\text { Wi-Fi }\end{array}$ & $3 \mathrm{G}$ \\
\hline
\end{tabular}

\subsection{Communication Standards comparison}

In the health monitoring system various technical communication standards are followed which are suitable for the transfer of data. Here are the some of the examples that are used in the domain of health care.

\subsubsection{Bluetooth Low Energy}

Operation Bandwidth: $2.4 \mathrm{Ghz}$

Topology: Star

Range: $150 \mathrm{~m}$

Data Rate: 1 Mbps

Features: 128-AES Encryption secure pairing prior to key sharing two keys used to provide authentication and privacy protection

Suitability for Health care: High

\subsubsection{Bluetooth Low Energy}

Operation Bandwidth: $2.4 \mathrm{GHz}$

Topology: Mesh

Range: $30 \mathrm{~m}$

Data Rate: $250 \mathrm{Kbps}$

Features: 128-AES Encryption. Network key shared towards network. Providing link key to secure application layer communication

Suitability for Health care: Moderate

\section{IOT TESTING FRAMEWORK}

This type of testing framework is created to validate the IOT components. Today there is huge need to deliver quality and faster services. There is an increasing need to access, create, use and share data from any device. The fact behind is to provide better process and control, over various interconnected IOT sensors. Hence, IOT testing process is essential. 


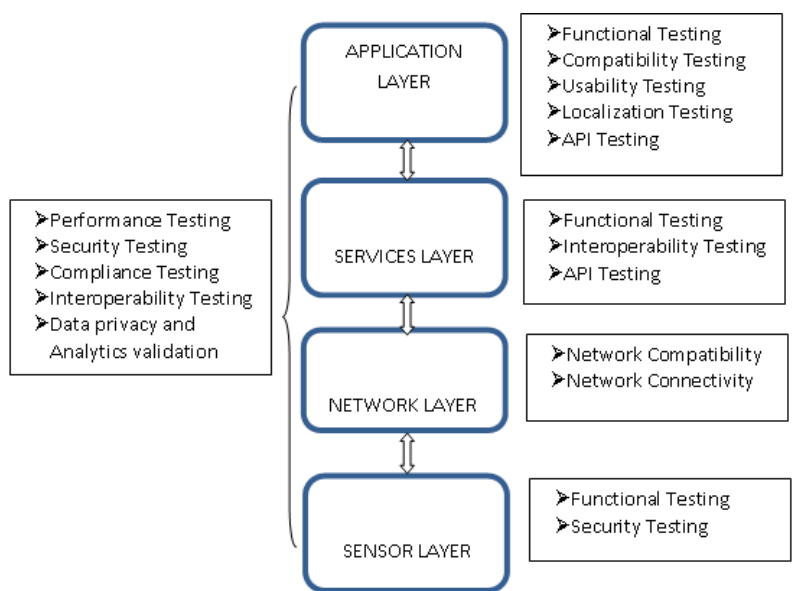

Figure 2: IoT Testing Framework

\section{A. Application Layer}

The application layer consists of functional testing and compatibility testing.

\section{B. Functional Testing}

The important concept for IoT functional testing is the process of testing in real-life environment using real devices or sensors to test those key components. The IoT smart technologies continue to increase enormously, and every test case differs as far as the functional core components are concerned [4].

\section{Compatibility Testing}

Compatibility testing should be a major priority in case of software development project. The complexity of Compatibility Testing will be more for QA teams in an IoT world since they will need to expand their scope of coverage to include various connected devices.

\section{Interoperability Testing}

Interconnectivity between devices ensures increased productivity at home or within industrial settings. This type of testing starts at a place, as the popularity rises and the number of devices and networks increases, the lack of interoperability between them becomes an issue.

\section{E. API Testing}

The main testing mechanism is the Application Programming Interface. API Testing is an essential process to achieve Quality Assurance. An interconnected world dependent on proprietary based business networks is highly reliable on the ability of these systems to exchange data.

\section{F. Network Compatibility Testing}

In Network compatibility testing, the team evaluates the various factors of a system in a network with varying parameters, such as operation bandwidth, performance evaluation, Network capacity, etc. In addition to that, it validates the application in different networks with the assistance of these attributes.

\section{G. Network Connectivity Testing}

To perform the network connectivity process, the accessibility of a remote system or test the connection to a port on a remote system can be tested.

- Testing availability of remote servers

- Testing TCP connectivity

- Sending test messages.

\subsection{Sensor Layer}

\section{H. Performance Testing}

This type of testing involves the speed of the network model through communication. It also includes the internal process of the software system that is embedded internally. IoT performance testing is necessary to be carry out through the Gateway and Network connectivity (protocols like HTTP, CoAP, MQTT etc), System Level (cloud Database, data processing, Cloud server), and the User Application Level. For instance: Check the throughput time against the standard time that defines the status conditions of network connectivity.

\section{Security Testing}

Security testing includes the factors like data authentication/authorization, data privacy, device identity, encryption/decryption, etc. In an IoT testing framework, security testing integrates both the device protection and the connectivity of network or connected cloud services.

\section{V.TEST MODEL IMPLEMENTATION}

The proposed method is the implementation of test model for internet of things. This model is divided into four categories. They are the Data Source, Data Gathering, and Data Transmission, Process Information and Cloud or Data center.

The model or design of an IoT domain can, generally speaking, be categorized and grouped according to their properties. Because the test of IoT systems is often closely related to these features, it is useful to devise a prototype that allows representing the plurality of devices and their characteristics within an IoT application domain [5]. By classifying the various core components of an IoT system and identifying each device in terms of its attributes, it is feasible to identify which functionality must be tested and to opt for which test(s) pattern(s) should be implemented accordingly.

\section{A. Data source}

It could represent some physical object such as devices, Machines, people, Tools, Vehicles etc Example: The objects can turn off the light and adjust the range of temperature inside the room

\section{B. Data Gathering}

This represents the sensors and actuators. Sensors have the ability to convert the information obtained in the outer world into data for analysis [6].

The main purpose of sensors is to gather data from the surrounding environment. Sensors and 'devices' of the IoT 
system form the front end. These actuators are connected directly or indirectly to the networks after signal conversion and processing.

\section{Network Connectivity}

The actuators or sensors will typically be connected to an HTTP network across the Internet worldwide. The Commercial IoT applications are communicated and the information is forwarded through Bluetooth or Ethernet (wired or wireless).

\section{Communication protocol}

The communication protocol is mainly implemented to enable simple, integrated devices to combine IoT through suitable networks having low bandwidth availability [7]. This protocol is mainly applied for machine-to-machine (M2M) communication and is specifically used for IoT devices that are completely depends upon IP protocols.

\section{E. Applications}

The data visualization is supported by the external applications. The data can be a real time or a stored historic database, and the triggering of alarms (either each time the condition is satisfied, or each time there is a variation in the condition).

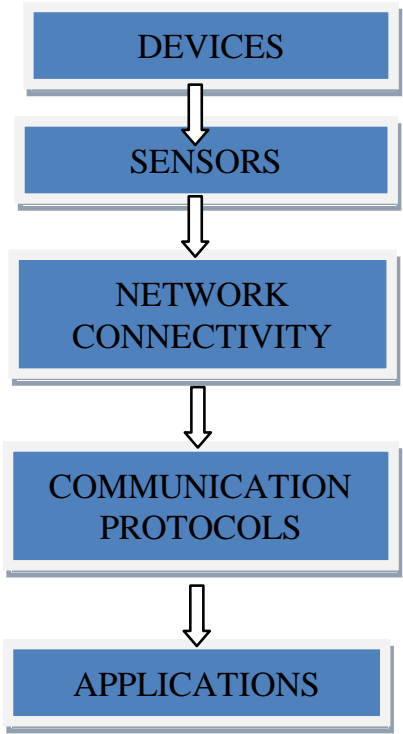

\section{Figure 3: Test Model for IOT Applications}

\section{IoT TEST PATTERN}

Considering the instance of test patterns in an IoT domain, it is possible to determine the strategies of testing that specifies the behaviors of an IoT system that described the test patterns [8].

\begin{tabular}{cc}
\hline TestPattern & \multicolumn{1}{c}{ Description } \\
\hline $\begin{array}{l}\text { Test Condition } \\
\text { Test Activity }\end{array}$ & $\begin{array}{l}\text { Checks the status of the sensors } \\
\text { Checks the measurement levels } \\
\text { are under the threshold values }\end{array}$ \\
Test Connectivity $\begin{array}{c}\text { Checks whether the links are } \\
\text { connected between the devices } \\
\text { The System should not only give } \\
\text { notifications, error messages and }\end{array}$
\end{tabular}

Test Service

Test Security

warnings

Checks whether the service is provided with standard protocols

Checks whether the sensor

Networks are located in placed

that is not accessible by the attackers

\section{TEST CASE GENERATION}

\subsection{Validation}

\subsubsection{Functionality}

In accordance to the scenario described, the system implements the following functionality:

- Monitoring and maintaining a personal record of health parameters, namely heart rate, blood pressure, and weight;

- Monitoring ambient conditions, specifically temperature, humidity, air quality and luminosity[9];

- Issuing alerts when the parameters being monitored indicate a problem: elevated heart rate

The alerts generated reflect the heart rate intervals defined in the following table. The actual intervals are calculated - in beats per minute (BPM) — using an estimated maximum heart rate [10] (MHR), following the stipulated by the American Heart Association (AHA)

Table 4: Categorization of heart rate for different intervals

\begin{tabular}{|l|l|}
\hline \multicolumn{1}{|c|}{ Category } & \multicolumn{1}{c|}{ Interval } \\
\hline Normal & $<50 \%$ \\
\hline Elevated & $51 \%-69 \%$ \\
\hline High & $70 \%-84 \%$ \\
\hline Very High & $85 \%$ \\
\hline
\end{tabular}

Various alerts were set for blood pressure values considered to be abnormal, according to the intervals defined by the AHA

Table 5: Categorization of blood pressure levels

\begin{tabular}{|l|l|l|}
\hline \multicolumn{1}{|c|}{ Category } & \multicolumn{1}{c|}{ upper value } & \multicolumn{1}{c|}{ lower value } \\
\hline Normal Values & $<119$ & And $<79$ \\
\hline Prehypertension & $120-138$ & or $80-88$ \\
\hline $\begin{array}{l}\text { Stage1Higher Blood } \\
\text { Pressure }\end{array}$ & $140-158$ & or $90-98$ \\
\hline $\begin{array}{l}\text { Stage 2 Higher Blood } \\
\text { Pressure }\end{array}$ & 160 or higher & $\begin{array}{l}\text { or } 100 \text { or } \\
\text { higher }\end{array}$ \\
\hline Hypertensive Crisis & $\begin{array}{l}\text { Higher than } \\
180\end{array}$ & $\begin{array}{l}\text { Higher than } \\
110\end{array}$ \\
\hline
\end{tabular}

\section{CONCLUSION AND FUTURE WORK}

The IoT represents the major and significant domain in the current technology development, related work addresses the key issues and challenges faced in IoT testing and the technologies used in various application scenarios.

The proposed paper provides a 
new testing approach transforming standards based IoT testing into a testing model and framework that enables scalable, distributed and automated IoT testing.

Test case generation for various aspects in incorporating test cases covering various factors such as usability, scalability, network connectivity and internet protocols is a difficult task due to the distributed and heterogenous nature of IoT systems Since the IoT devices are made of different hardware and technologies so there are always difficulties in testing them. There are many critical bugs related to functionality, performance, and security. Since IOT is evolving at a faster rate so the quality of the software in IoT devices cannot be compromised. The users of the connected devices are unaware of how the IoT system is working but are very much accustomed to using IoT technology. Even though there are several tools available that can be used in the validation of IoT components, there occurs number of issues that can be addressed: a technological review of existing solutions reveals the lack of a comprehensive test solution for automated integration testing. Focusing on a specific protocol, network, or standard, decreasing the feasibility of upgrading or extension, and not providing out-of-the-box functionality are among the most common shortcomings detected. Alerts are triggered when the patient's status is validated to require immediate attention and pre-set operations are generated by the variations in the abnormal conditions.

In future Artificial Intelligence and machine learning techniques can be integrated with testing framework which can be used in software testing phases. Each phase can be implemented with AI methods like Neural Networks, CBR and Intelligence planning or statistical concepts such as Regression modeling and PCA. Thus these approaches for automatic testing have significant effect in decreasing testing costs and improving the software quality.

\section{REFERENCES}

1. Vandana Sharma, Ravi Tiwari, A review paper on "IOT" \& It's Smart Applications, International Journal of Science, Engineering and Technology Research (IJSETR), Volume 5, Issue 2, February 2016

2. Zainab H. Ali, Hesham A. Ali, Mahmoud M. Badawy, Internet of Things (IoT): Definitions, Challenges and Recent Research Directions, International Journal of Computer Applications (0975 - 8887) Volume 128 - No.1, October 2015.

3. Software Testing Techniques in IoT, Ghadeer Murad; Aalaa Badarneh; Abdallah Quscf; Fadi Almasalha 2018 8th International Conference on Computer Science and Information Technology (CSIT) 11 October 2018, IEEE

4. John Esquiagola, Laisa Costa, Pablo Calcina, Geovane Fedrecheski and Marcelo Zuffo, Performance Testing of an Internet of Things Platform DOI: $10.5220 / 0006304503090314$ In Proceedings of the 2nd International Conference on Internet of Things, Big Data and Security (IoTBDS 2017), pages 309-314 ISBN: 978-989-758-245-5

5. Bestoun S. Ahmed*, Miroslav Bures, Karel Frajtak, and Tomas Cerny, Aspects of Quality in Internet of Things (IoT) Solutions: A Systematic Mapping Study,

6. Pramod Mathew Jacob, Prasanna Mani,A Reference Model For Testing Internet Of Things Based Applications, Journal of Engineering Science and Technology Vol. 13, No. 8 (2018) 2504 - 2519

7. Ghadeer Murad,Aalaa Badarneh, Abdallah Quscf,Fadi Almasalha, Software Testing Techniques in IoT 2018 8th International Conference on Computer Science and Information Technology (CSIT)

8. Joao Pascoal Faria, Bruno Lima A pattern-based IoT testing framework, Companion Proceedings for the ISSTA/ECOOP 2018 Workshops Pages 125-131

9. Shubham Banka, Isha Madan and S.S. Saranya,
Smart Healthcare Monitoring using IoT,International Journal of Applied Engineering Research ISSN 0973-4562 Volume 13, Number 15 (2018) pp.

10. C.Senthamilarasi,J.JansiRani,B.Vidhya,.H.Aritha Smart Patient Health Monitoring System Using IoT,International Journal of Pure and Applied Mathematics Volume 119 No. 16 2018, 59-70

\section{AUTHORS PROFILE}

V.Sathyavathy Ph.D Research scholar, Karpagam Academy of Higher Education

Dr.D.Shanmuga Priyaa Professor,Department of CS,CA\&IT. Karpagam Academy of Higher Education 